\title{
Alterações das Pressões Anais em Pacientes Constipados por Defecação Obstruída
}

\author{
Anal Pressure Changes in Patients with Outlet Constipation
}

\author{
MARIA AUXILIADORA PROLUNGATTI CÉSAR ${ }^{1}$; WILMAR ARTUR KLUG²; HELLY ANGELA CARAM AGUIDA; \\ JORGE ALBERTO ORTIZ ${ }^{4}$; CHIA BIN FANG ${ }^{5}$; PERETZ CAPELHUCHNIK ${ }^{6}$ \\ ${ }^{1}$ Doutora em Cirurgia; ${ }^{2}$ Professor Titular; ${ }^{3}$ Mestre em Cirurgia; ${ }^{4}$ Mestre em Cirurgia; \\ ${ }^{5}$ Professor Adjunto; ${ }^{6}$ Professor Titular.
}

CÉSAR MAP; KLUG WA; AGUIDA HAC; ORTIZ JA; FANG CB; CAPELHUCHNIK P. Alterações das Pressões Anais em Pacientes Constipados por Defecação Obstruída. Rev bras Coloproct, 2008;28(4): 402-408.

RESUMO: Introdução: a constipação é um sintoma de doença multifatorial. O diagnóstico correto é importante para orientar a terapêutica. Nas formas de defecação obstruída há vários fatores relacionados como gênero, idade, hábitos, paridade, doenças associadas e distúrbios específicos da evacuação. Entre os métodos para diagnóstico a manometria é usada pela facilidade técnica e disponibilidade. Objetivo: verificar o valor da manometria isoladamente em constipados por defecação obstruída. Método: examinamos quarenta pacientes do Ambulatório de Coloproctologia da Santa Casa de São Paulo com diagnóstico de defecação obstruída. As medidas de pressão retal e anal foram comparadas com um grupo controle de 60 indivíduos considerados normais do ponto de vista proctológico. Separados os pacientes consoante a causa da constipação, verificou-se o valor do método manométrico em cada causa específica. Resultados: houve somente diferenças entre as medidas de pressão retal e anal em repouso e pressão máxima de contração entre os normais e os vários tipos de constipados, mas não diferenças específicas entre as várias modalidades de constipação. Conclusão: os vários métodos de fisiologia anal são importantes e necessários em conjunto para o diagnóstico correto. A manometria contribui para a investigação dos distúrbios funcionais, devendo sempre ser incluída. Contudo, seu valor como método isolado é questionável.

Descritores: constipação, defecação obstruída, manometria, pressão anal.

\section{INTRODUÇÃO}

Constipação é sintoma comum, mais freqüente em mulheres, incidindo de preferência nos idosos. Foram já estabelecidos firmes critérios que facilitam o diagnóstico, que incluem: ritmo intestinal com menos de três evacuações por semana, sensação de dificuldade para evacuar, fezes pequenas e endurecidas e sensação de evacuação incompleta. Considera-se constipado aquele que apresentar dois ou mais desses sin-

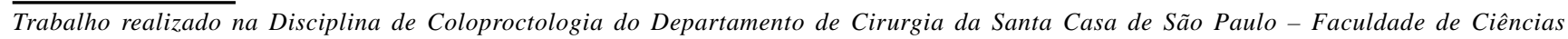
Médicas. 
tomas durante pelo menos três meses ao longo do ano. O diagnóstico de constipação depende na maioria das vezes da percepção individual, já que o que é normal para alguns pacientes não o é para outros. Muitos dos que se dizem constipados não se encaixam nas definições específicas ${ }^{1}$. Os sintomas podem ocorrer secundariamente a várias doenças e mecanismos, varia de leve e temporária a grave e crônica, interferindo no estilo de vida. Fatores psicológicos e sociais, ansiedade e depressão, podem estar envolvidos ${ }^{2}$. Gravidez e paridade são fatores importantes ${ }^{3}$.A avaliação dos pacientes exige minuciosa apreciação da história, exame físico, proctológico, ginecológico, além de estudos de fisiologia anal, mas muitas vezes pouca atenção é dada às inúmeras circunstâncias envolvidas. A atitude geral é considerá-los um grupo comum e tratá-los de modo simplista. Poucos são encaminhados ao especialista, sendo que o envio decorre do fracasso das tentativas anteriores de tratamento.

Há dois tipos fundamentais de constipação e sua caracterização é importante para tratar de modo adequado: trânsito lento e defecação obstruída. Indivíduos com trânsito lento do cólon beneficiam-se das fibras e laxantes, ao passo que os com defecação obstruída geralmente não necessitam de medicação, mas reposição de fibras e treinamento funcional do diafragma pélvico ${ }^{4}$. A identificação dos distúrbios funcionais facilita a terapêutica e são realizados pelos métodos de fisiologia anal: testes de sensibilidade, manometria, teste de expulsão do balão, eletromiografia, tempo de latência do nervo pudendo, proctografia e marcadores de trânsito do cólon ${ }^{5}$. Por estes meios é possível separar as causas e classificar o tipo de distúrbio, ressaltando a constipação por defecação obstruída, mais freqüente que a inércia do cólon. $\mathrm{O}$ uso desses métodos deve ser feito de forma racional. Entre todos ressalta pela simplicidade, facilidade de execução e disponibilidade o exame de manometria anal.

Quando se está diante de um paciente constipado, fica clara a necessidade de uma avaliação global, incluindo os inúmeros aspectos já citados. Selecionados doentes com defecação obstruída, o valor isolado da manometria anal é objeto de discussão, pois seu propósito é medir especificamente as alterações da pressão anal, desconsiderando outras informações a partir dos demais métodos. Portanto, o objetivo deste trabalho foi examinar pacientes com defecação obstruída pela manometria, isoladamente, comparando-os com um grupo de normais, para medir seu valor e sua contribuição para o diagnóstico.

\section{CASUÍSTICA E MÉTODO}

Foram estudados 40 constipados por defecação obstruída, sendo 31 mulheres e nove homens, com idade média de 52,28 $\pm 14,23$ anos, variando entre 18 e 72 anos. Os pacientes vieram do Ambulatório de Coloproctologia da Santa Casa de Misericórdia de São Paulo - Departamento de Cirurgia da Faculdade de Ciências Médicas. Deles foram colhidos identificação, história clínica, idade, gênero, paridade e tipo de parto, cirurgias do períneo anteriores e outros fatores relacionados.

\section{EXAME DE MANOMETRIA RETAL E ANAL}

Para manometria foi utilizado o aparelho Proctosystem PL-3000, dotado de balão conectado a transdutor de membrana que gera sinal elétrico, registrado em forma digital e gráfica. Os exames iniciaram com introdução o balão no reto, onde foram medidas as pressões de repouso e defecação. No ânus, com o balão posicionado na zona de maior pressão, foram medidas as pressões de repouso, contração e menor pressão de defecação. As manometrias foram comparadas a um grupo controle existente, composto de 60 pacientes sem doença ano-retal, com idade média semelhante $(49,70 \pm 17,99)$, examinado com o mesmo equipamento e técnica ${ }^{6}$. A análise estatística se fez pelo teste $t$ de Student para comparação de médias, controlado pelo teste de Levene para igualdade das variâncias.

\section{RESULTADOS}

Na manometria não houve variação estatisticamente significante entre os grupos em relação à idade $(52,28 \pm 14,83$ versus $49,70 \pm 17,99)$. Houve diferença nas pressões máximas de repouso e contração anal, tendo os constipados valores menores que o controle. Não existiram diferenças significantes entre os dois grupos em relação à pressão mínima de evacuação, pressão retal de evacuação e extensão da zona de hiperpressão (Tabela 1). 
Tabela 1 - Manometria retal e anal em pacientes constipados comparados com grupo controle.

\begin{tabular}{lrrc}
\hline Exames & Constipados & Controle & p \\
\hline Pressão anal máxima de repouso & $61,45 \pm 18,42$ & $71,30 \pm 25,39$ & $\mathrm{p}<0,05$ \\
Pressão anal máxima de contração & $132,75 \pm 52,84$ & $209,45 \pm 108,46$ & $\mathrm{p}<0,05$ \\
Pressão anal mínima de evacuação & $34,03 \pm 12,41$ & $37,83 \pm 16,28$ & $\mathrm{Ns}$ \\
Pressão retal & $13,35 \pm 12,55$ & $19,60 \pm 9,42$ & $\mathrm{p}<0,05$ \\
Pressão retal de evacuação & $56,78 \pm 21,82$ & $80,02 \pm 32,45$ & $\mathrm{Ns}$ \\
Zona de hiperpressão $(\mathrm{cm})$ & $3,23 \pm 0,83$ & $3,28 \pm 0,74$ & $\mathrm{Ns}$ \\
\hline
\end{tabular}

\section{DIAGNÓSTICOS E OS EXAMES NO LABORATÓRIO DE FISIOLOGIAANAL}

Os doentes foram separados consoante o diagnóstico final obtido no laboratório, para melhor avaliar o valor e significado da manometria nos diversos grupos.

\section{RETOCELE}

Havia retocele em $45 \%$ do total (18 dos 40). Em quatro dos $18(22,22 \%)$ foi única causa de constipação. A contração paradoxal do pubo-retal associouse à retocele em 44,4\%, a invaginação interna em $27,7 \%$ e sigmoidocele em $22,22 \%$. Noa demais não houve causas associadas. Os pacientes com retocele, independentemente das associações, foram comparados com os 22 que não a apresentavam. Não houve diferenças estatisticamente significantes em relação a ritmo intestinal com a utilização ou não de laxantes, duração da queixa, paridade e idade. Através da manometria foi observado que as médias das pressões de repouso, contração e evacuação foram maiores no grupo retocele em comparação aos demais, mas sem diferença estatisticamente significante (Tabela 2).

\section{CONTRAÇÃO PARADOXAL DO PUBO- RETAL}

O diagnóstico de contração paradoxal do puboretal já fora considerado a partir da atividade mioelétrica, diagnóstico feito em $47,5 \%$ (19 pacientes) e também por proctografia em $42,1 \%$ deles. Na comparação dos pacientes com contração paradoxal e os sem esta alteração, não houve diferenças em relação a ritmo intestinal com a utilização ou não de laxantes, duração da queixa, paridade e idade. Do ponto de vista da manometria, porém, não observamos as alterações manométricas esperadas, em especial o relaxamento ao evacuar (Tabela 3).

\section{SIGMOIDOCELE}

Foi diagnosticada através da proctografia em $15 \%$ (6 pacientes). Os portadores da afecção e os outros não apresentaram diferenças estatisticamente significantes em relação ao ritmo intestinal com a utilização ou não de laxantes, duração da queixa, paridade e idade. Comparando pacientes portadores ou não de sigmoidocele pela manometria não se encontraram diferenças estatisticamente significantes (Tabela 4).

Tabela 2 - Médias de pressões anais e retais em constipados portadores ou não de retocele.

\begin{tabular}{lrrc}
\hline Manometria & Retocele & Sem retocele & p \\
\hline Máxima pressão de repouso & $66,61 \pm 17,84$ & $57,23 \pm 18,19$ & Ns \\
Máxima pressão de contração & $142,94 \pm 63,20$ & $124,41 \pm 42,31$ & Ns \\
Mínima pressão de evacuação & $34,78 \pm 13,53$ & $33,41 \pm 11,69$ & Ns \\
Pressão retal & $11,83 \pm 16,14$ & $14,59 \pm 8,83$ & Ns \\
Pressão retal de evacuação & $62,5 \pm 25,54$ & $52,09 \pm 17,46$ & Ns \\
Zona de hiperpressão & $3,06 \pm 0,94$ & $3,36 \pm 0,73$ & Ns \\
\hline
\end{tabular}


Tabela 3 - Médias de pressões anais e retais em constipados portadores ou não de contração paradoxal do pubo-retal.

\begin{tabular}{lccc}
\hline Manometria ano-retal & $\begin{array}{c}\text { Presença de contração } \\
\text { paradoxal do pubo-retal }\end{array}$ & $\begin{array}{c}\text { Sem contração paradoxal } \\
\text { do pubo-retal }\end{array}$ & P \\
\hline Pressão máxima de repouso & $62,79 \pm 18,01$ & $60,24 \pm 19,14$ & $\mathrm{Ns}$ \\
Pressão máxima de contração & $127,10 \pm 49,33$ & $137,90 \pm 56,54$ & $\mathrm{Ns}$ \\
Pressão mínima de evacuação & $37,58 \pm 11,83$ & $30,81 \pm 12,3$ & $\mathrm{Ns}$ \\
Pressão retal de repouso & $13,16 \pm 8,96$ & $13,52 \pm 15,32$ & $\mathrm{Ns}$ \\
Pressão retal de evacuação & $56,63 \pm 21,06$ & $56,90 \pm 23,00$ & $\mathrm{Ns}$ \\
Zona de hiperpressão & $2,99 \pm 0,77$ & $3,08 \pm 0,72$ & $\mathrm{Ns}$ \\
\hline
\end{tabular}

\section{INVAGINAÇÃO INTERNA}

Diagnosticada antes pela proctografia $25 \%(10$ pacientes). Quarenta por cento das pacientes eram nulíparas. Os grupos com e sem invaginação foram comparados. Não houve diferenças em relação a ritmo intestinal com ou sem laxantes, duração da queixa, paridade e idade. Pela manometria não se observou nenhuma diferença estatisticamente significante (Tabela 5).

Comparamos os resultados separando os doentes por diagnóstico, incluindo em colunas todos os pacientes com determinado distúrbio, de forma que pacientes com dois ou mais apareceram mais de uma vez, na coluna correspondente. Agrupando assim, a manometria não evidenciou diferença nas pressões, exceto quando comparamos cada um deste grupo de diagnósticos em relação ao grupo controle na pressão máxima de contração, pressão retal em repouso e evacuação (Tabela 6).

\section{DISCUSSÃO}

A constipação intestinal, tanto em sua modalidade inércia quanto por defecação obstruída é com-

Tabela 4 - Médias de pressões anais e retais em constipados portadores ou não de sigmoidocele.

\begin{tabular}{lccc}
\hline Manometria ano-retal & Presença de sigmoidocele & Sem sigmoidocele & p \\
\hline Pressão máxima de repouso & $61,50 \pm 11,06$ & $61,44 \pm 19,56$ & Ns \\
Pressão máxima de contração & $143,00 \pm 42,31$ & $130,90 \pm 54,83$ & Ns \\
Pressão mínima de evacuação & $31,50 \pm 12,24$ & $34,47 \pm 12,56$ & Ns \\
Pressão retal de repouso & $9,67 \pm 8,29$ & $14,00 \pm 13,14$ & Ns \\
Pressão retal de evacuação & $66,50 \pm 28,01$ & $55,06 \pm 20,58$ & Ns \\
Zona de hiperpressão & $3,33 \pm 0,93$ & $2,99 \pm 0,70$ & Ns \\
\hline
\end{tabular}

Tabela 5 - Médias das pressões anais e retais em constipados portadores ou não de invaginação interna.

\begin{tabular}{lccc}
\hline Manometria ano-retal & Presença de invaginação & Sem invaginação & p \\
\hline Pressão máxima de repouso & $61,00 \pm 14,48$ & $60,44 \pm 19,75$ & $\mathrm{Ns}$ \\
Pressão máxima de contração & $143,80 \pm 51,13$ & $124,80 \pm 54,61$ & $\mathrm{Ns}$ \\
Pressão mínima de evacuação & $28,90 \pm 9,44$ & $36,09 \pm 12,77$ & $\mathrm{Ns}$ \\
Pressão retal & $9,20 \pm 6,84$ & $14,69 \pm 13,50$ & $\mathrm{Ns}$ \\
Pressão retal de evacuação & $54,01 \pm 24,88$ & $56,25 \pm 21,15$ & $\mathrm{Ns}$ \\
Zona de hiperpressão & $3,01 \pm 0,86$ & $3,09 \pm 0,71$ & $\mathrm{Ns}$ \\
\hline
\end{tabular}


Tabela 6 - Valores de manometria nos diferentes grupos diagnósticos.

\begin{tabular}{|c|c|c|c|c|c|c|}
\hline Manometria & Retocele & $\begin{array}{c}\text { Contração } \\
\text { paradoxal do } \\
\text { do pubo-retal }\end{array}$ & Invaginação & Sigmoidocele & Controle & $\mathbf{p}$ \\
\hline $\begin{array}{l}\text { Pressão máxima } \\
\text { de repouso }\end{array}$ & $66,61 \pm 17,84$ & $62,79 \pm 18,01$ & $61,00 \pm 14,48$ & $61,50 \pm 11,06$ & $71,30 \pm 25,39$ & Ns \\
\hline $\begin{array}{l}\text { Pressão máxima } \\
\text { de contração }\end{array}$ & $142,90 \pm 63,2^{*}$ & $127,10 \pm 49,33^{*}$ & $143,80 \pm 51,13$ & $143,00 \pm 42,31$ & $209,45 \pm 108,46$ & $\mathrm{p}<0,05$ \\
\hline $\begin{array}{l}\text { Pressão mínima } \\
\text { de evacuação }\end{array}$ & $34,78 \pm 13,53$ & $37,58 \pm 11,83$ & $28,90 \pm 9,44$ & $31,50 \pm 12,24$ & $37,83 \pm 16,28$ & Ns \\
\hline Pressão retal & $11,83 \pm 16,14 *$ & $13,16 \pm 8,96$ & $9,20 \pm 6,84^{*}$ & $9,67 \pm 8,29$ & $19,60 \pm 9,42$ & $\mathrm{p}<0,05$ \\
\hline $\begin{array}{l}\text { Pressão retal } \\
\text { de evacuação }\end{array}$ & $62,5 \pm 25,54^{*}$ & $56,63 \pm 21,06^{*}$ & $54,01 \pm 24,88$ & $66,50 \pm 28,01$ & $80,02 \pm 32,45$ & $\mathrm{p}<0,05$ \\
\hline
\end{tabular}

plexa em sua gênese e difícil de explicar em forma simplista. É multifatorial, incluindo aspectos dietéticos, idade, condições gerais de saúde, hormônios e polipeptídios intestinais, paridade, lesões nervosas e mudanças na fisiologia dos órgãos pélvicos. Na inércia há diminuição da concentração plasmática de motilina e influência da progesterona na gravidez ${ }^{7}$. Parece haver também um papel para a somatostatina ${ }^{8}$. Outros fatores devem ser considerados na gênese ou agravamento dos sintomas. Entre eles o programa alimentar, nível de atividade física, ingestão de água ${ }^{9}$, quantidade adequada de fibras ${ }^{10}$, embora alguns com constipação grave possam piorar os sintomas ingerindo fibras em excesso ${ }^{11}$. Além disso, em vista do aumentado tempo de trânsito há mais absorção de água e isso resseca as fezes. Na defecação obstruída predominam os fenômenos relacionados com a fisiologia da evacuação, fatores anatômicos e funcionais pélvicos e lesões nervosas.

Em relação a manometria, ao compararmos indivíduos constipados pelas várias causas aos normais, os valores observados mostraram muitas diferenças. De um modo geral, os níveis de pressão anal à contração e retal à evacuação foram significantemente menores. Isto sugere uma competência funcional maior nos pacientes do grupo controle. Era também esperado que pacientes com evacuação obstruída, em especial por contração paradoxal, apresentassem valores da pressão mínima na evacuação mais altas que o grupo controle, o que não foi encontrado. A expectativa é justificada, pois $47,50 \%$ deles foram diagnosticados como portadores de contração paradoxal do pubo-retal, o que deveria se traduzir por esta maior pressão mínima de evacuação. Não fica clara a razão da falha em demonstrar a alteração. Deve ser ressaltado que o esforço para a evacuação em laboratório é diverso daquele obtido na intimidade. Por estas razões a própria existência da contração paradoxal do pubo-retal é posta em discussão ${ }^{12}$. Na experiência de quem examina, fica clara a necessidade dos exames serem feitos em ambiente e condições propícias. Sendo a maioria mulheres, o recato, o medo diante do equipamento, presença de estranhos ao convívio e ambiente opressor de um hospital certamente interfere. Quanto às pressões anais de repouso e contração e retal de repouso, houve diferença não significante entre os grupos de constipados e controle, a menor para os pacientes constipados. Uma das razões para as diferenças poderia ser a própria disparidade do grupo controle; outra o dano provocado pelo esforço repetitivo na inervação do diafragma pélvico. De fato, observou-se que vários fatores, incluindo sexo e idade, podem interferir nos valores médios de pressão anal, inclusive em pacientes não portadores de doença proctológica ${ }^{6}$. Verificamos discreta variação na pressão máxima de repouso que, embora significante, pode ser imputada às variações técnicas e de observador. Contudo, foi notável a queda dos valores de pressão anal máxima de contração, menor nos constipados que em normais. Há que se invocar, para explicar a diferença, alterações anatômicas e funcionais sofridas pelo esfíncter externo do ânus no decorrer de anos de constipação com esforços repetidos, paridade, sexo feminino, diversos tipos de manipulação, uso de supositórios e enemas. 
Com base teórica, poder-se-ia supor a existência e desencadeamento de um complexo: esforço de evacuação repetido causando dano na inervação, descida do diafragma pélvico ${ }^{13} \mathrm{e}$ hipotonia, como resultante final da constipação. Portanto, acompanhamento destes pacientes por prazo longo permitiria esta avaliação.

Os resultados da manometria dependem de resposta subjetiva e cooperação ${ }^{14}$. Os movimentos da sonda no canal anal podem induzir contrações involuntárias do esfíncter anal externo, o que contribui para as variações encontradas. Isso é importante principalmente quando se compara a manometria de perfusão e a por balão, esta última menos sujeita às variações, já que é exame mais estático. Em relação à pressão de canal anal, pacientes com defecação obstruída tiveram pressões basais menores que o grupo controle ${ }^{15}$. Neste estudo não houve diferenças entre as pressões de repouso, e evacuação em relação ao controle e entre os grupos entre si. Se não existe esta diferença pode-se questionar a importância da manometria no diagnóstico diferencial dos tipos de constipação intestinal. Seria de esperar que pacientes com contração paradoxal do pubo-retal tivessem a pressão de evacuação do canal anal aumentada em relação ao grupo controle. A falta desse dado levaria a considerar a manometria de menor importância no diagnóstico da constipação, pois não foi importante como método diagnóstico específico ${ }^{1}$.

\section{CONCLUSÃO}

A constipação intestinal é doença multifatorial, sendo fundamental diagnóstico preciso para orientar a terapêutica correta. Os vários exames de fisiologia anal são importantes e a manometria anal contribui para a investigação do distúrbio, devendo sempre ser incluída na avaliação, mas seu valor como método isolado é questionável.

\begin{abstract}
Background: constipation is a complex problem and precise diagnosis is required for adequate therapy. When treating patients with obstructed defecation, many factors as gender, age, personal habits, childbirth, associated diseases and other specific pelvic disorders must be considered. Manometry is the preferred diagnosis method due to its simplicity and general availability. Objective: the aim of this work was to determine rectal and anal pressures in patients with outlet constipation. Method: forty patients diagnosed with outlet constipation were examined using manometry by Coloproctology Ambulatory of Santa Casa of São Paulo. The results were compared with a control group of 60 normal. Rectal and anal pressures were measured by ballon manometry, with the patients grouped by type of constipation. Results: we observed alterations in rectal and anal resting and anal squeese pressures in constipated individuals, but no pressure differences between the various types of constipation. Conclusion: the different diagnostic methods are relevant for a correct diagnosis. Although baloon manometry should be one of these procedures, it should not be used as the only diagnosis method.
\end{abstract}

Keywords: constipation, outlet constipation, manometry, anal pressure.

\section{REFERÊNCIAS}

1. Ashraf W; Park F; Lof J; Quigley EM. An examination of the reliability of reported stool frequency in the diagnosis of idiopathic constipation. Am J Gastroenterol, 1996; 91(1): 2632.

2. Mellgreen A; Anzen B; Nilsson BY; Johansson C; Dolk A; Gillgren P; Bremmer S; Holmstrom B. Results of rectocele repair- a prospective study. Dis Colon rectum , 1995; 38(1): 7-13.

3. Klug WK; Ortiz JA; Aguida HAC; Fang CB; Capelhuchnik P. Alteração das pressões anais na gravidez. Rev bras Coloproct, 2007; 27(2): 196-201.
4. Camilleri M. Functional gastrointestinaldisorders: novel insights and treatments. Medscape Gastro J, 1999; 1(1):1-13.

5. Cesar MAP. Diagnóstico da constipação intestinal por defecação obstruída através dos exames de fisiologia anal. São Paulo; 2000. Tese de mestrado. Faculdade de Ciências Médicas da Santa Casa de São Paulo.

6. Aguida, HAC. Efeito da idade e do sexo nas pressões do canal anal-estudo manométrico. São Paulo; 2000. Tese de mestrado. Faculdade de Ciências Médicas da Santa Casa de São Paulo.

7. Qiu XH; Yang MG; Pengg JJ; Huang YX; XU CF et al. Changes of plasma motilin concentration in pregnancy and early postpartum period. Sheng Li Xue Bao, 1994; 46(1): 100-104. 
8. Holst N; Jenssen TG; Burhol PG. Plasma concentrations of motilin and somatostatin are increased in late pregnancy and postpartum. Br J Obstet Gynaecol, 1992; 99(4): 338-341.

9. Derbyshire E; Davies J; Costarelli V; Dettmar P. Diet, physical inactivity and the prevalence of constipation throughout and after pregnancy. Matern Chil Nutr, 2006; 2(3): 127-134.

10. Jewell DJ; Young G. Interventions for treating constipation in pregnancy. Cochrane Database Syst Rev, 2001; (2): CD 001142.

11. Müller-Lissner SA; Kamm MA; Scarpignato C; Wald A. Myths and misconceptions about chronic constipation. Am J Gastroenterol, 2005; 100(1): 232-242.

12. Schouten WR; Briel JW; Auerda JJA; Vandam JH; Gosselink MJ; Ginai AZ; Hop WCJ. Anismus: facts or fiction ?. Dis Colon Rectum, 1997; 40: 1033-1041.
13. Ho YH; Goh HS. The neurophysiological significance of perineal descend. Int J Colorectal Dis, 1995; 10(2): 107-111.

14. Ryhammer AM; Laurberg S; Herrman AP. Test-repeatability of anorectal physiology tests in healthy volunteers. Dis Colon Rectum, 1996; 40: 287-292.

15. Bassoti G; Chiaroni G; Vantini I; Betti C; Fusaro C et al. Anorectal manometric abnormalities and colonic propulsive impairment in patients with severe chronic idiopathic constipation. Dig Dis Sci, 1994; 39(7): 1558-1564.

\section{Endereço para correspondência: \\ DR. WILMAR ARTUR KLUG}

Alameda Ribeirão Preto, no 487 - Ap. 103

São Paulo - SP - Cep 01331-001

E-mail: klug@doctor.com 